\title{
Higgs mass and noncommutative geometry
}

\author{
Andrzej Sitarz *† \\ Department of Field Theory \\ Institute of Physics \\ Jagiellonian University \\ Reymonta 4, 30-059 Kraków, Poland
}

\begin{abstract}
We show that the description of the electroweak interactions based on noncommutative geometry of a continuous and a discrete space gives no special relations between the Higgs mass and other parameters of the model. We prove that there exists a gauge invariant term, linear in the curvature, which is trivial in the standard differential geometry but nontrivial in the case of the discrete geometry. The relations could appear only if one neglects this term, otherwise one gets the Lagrangian of the Standard model with the exact number of free parameters.
\end{abstract}

*Partially supported by KBN grant 2 P302 1684

†E-mail: sitarz@if.uj.edu.pl 


\section{Introduction}

The noncommutative geometry has brought a remarkable geometric picture explaining the nature of the Higgs field and the symmetry breaking mechanism [1]-[6] in the Standard Model. The electroweak part of the model is explained as originating from the product of the continuous Minkowski space-time by a discrete two-point space.

The Higgs field is a connection, which arises from the geometry of the discrete space. The latter corresponds to the left and right fermions, with appropriate gauge groups associated with each point. The bosonic part of the action comes naturally as a Yang-Mills action, including the quartic Higgs potential term. The couplings of the Higgs field to fermions arise from the usual couplings between gauge potential and matter fields.

In this construction one recovers the Lagrangian of the Standard Model with one less free parameter. This has led to speculations on the possible theoretical predictions of the Higgs mass, even though they were established only on the classical level. However, recent results [7] suggest that they will not survive quantum corrections.

In this paper we shall advocate the idea that these relations cannot be maintained already on the classical level, due to the presence of a gauge-invariant term, linear in the field strength. This term, together with the Yang-Mills term, gives the original Lagrange function of the Standard Model, with the same number of parameters. Therefore, unless this term vanishes, there are no classical relations between the Higgs mass and other parameters of the Standard Model.

The existence of such term is characteristic for the discrete geometry, therefore our arguments do not change the relations obtained in some other approaches [8]-11].

\section{Gauge Theory on $M_{4} \times \mathbf{Z}_{2}$}

In this section we shall review the basic methods of constructing the gauge theory on a product of the continuous Minkowski space $M_{4}$ and the discrete two-point space $\mathbf{Z}_{2}$. We shall present here only the most substantial facts, more detailed account of the construction can be found in [1]-[1] and [12]. We restrict ourselves only to the bosonic sector, as it simplifies our considerations and does not influence the results.

Let $x^{\mu}$ denote the coordinates on $M_{4}$ and let $y= \pm$ label the points of $\mathbf{Z}_{2}$. The differentiation on the product space $M_{4} \times \mathbf{Z}_{2}$ has the following form. If $f$ is a function, then:

$$
d f=d x^{\mu}\left(\partial_{\mu} f\right)+\chi\left(\partial_{+} f\right),
$$

where $d x^{\mu}$ are from the basis of one-forms on $M_{4}$ and $\chi$ is an object denoting 
the one-form on $\mathbf{Z}_{2}$. The partial derivative $\partial_{+}$is defined as follows:

$$
\left(\partial_{+} f\right)(x, y)=f(x, y)-f(x,-y)
$$

The remaining rules of the differential calculus define the product of one forms:

$$
\begin{aligned}
d x^{\mu} \otimes d x^{\nu} & =-d x^{\nu} \otimes d x^{\mu}, \\
d x^{\mu} \otimes \chi & =-\chi \otimes d x^{\mu},
\end{aligned}
$$

the left multiplication by functions:

$$
\begin{aligned}
f(x, y) d x^{\mu} & =d x^{\mu} f(x, y), \\
f(x, y) \chi & =\chi f(x,-y),
\end{aligned}
$$

and the action of the external derivative $d$ on them:

$$
\begin{aligned}
d\left(d x^{\mu}\right) & =0 \\
d(\chi) & =2 \chi \otimes \chi
\end{aligned}
$$

The gauge potential A splits therefore into two parts, the vector part $d x^{\mu} A^{\mu}$ and the scalar part $\chi \Phi$. The names correspond to the behavior of the coefficients $A_{\mu}$ and $\Phi$ under Lorentz transformations in $M_{4}$. The unitarity of the gauge group enforces that $A=-A^{\star}$, thus, since $d x^{\mu}$ are selfadjoint and $\chi^{\star}=-\chi$, we obtain the following relations:

$$
\begin{aligned}
A_{\mu}(x, y) & =-A_{\mu}^{\dagger}(x, y), \\
\Phi(x, y) & =\Phi^{\dagger}(x,-y) .
\end{aligned}
$$

The curvature two-form $\mathbf{F}=d \mathbf{A}+\mathbf{A} \otimes \mathbf{A}$ splits into three terms:

$$
\mathbf{F}=d x^{\mu} \otimes d x^{\nu} F_{\mu \nu}+d x^{\mu} \otimes \chi \mathcal{F}_{\mu}+\chi \otimes \chi f
$$

where $F_{\mu \nu}$ is the usual curvature tensor (obtained independently for each value of $y), \mathcal{F}_{\mu}$ is a mixed term, which includes the gradient of $\Phi$ and the coupling between $A_{\mu}$ and $\Phi$. The last term, $f$, which depends only on $\Phi$ shall be of our primary interest, as it is responsible for the appearance of the Higgs' quartic potential in the Yang-Mills action.

The precise form of $f$ is:

$$
f(x, y)=\Phi(x, y)+\Phi(x,-y)+\Phi(x,-y) \Phi(x, y) .
$$

By introducing a shifted potential $\Psi(x, y)=1+\Phi(x, y)$ and using relation (10), we may rewrite (12) as:

$$
f(x, y)=\Psi^{\dagger}(x, y) \Psi(x, y)-1 .
$$


To construct the Yang-Mills action we need to have a metric $\eta$. We take it to be a functional from the cartesian square of the space of one-forms to the algebra of functions, which is linear with respect to addition and left- and rightmultiplication by functions. In the considered case we additionally postulate that the metric is diagonal, i.e., that $\eta\left(d x^{\mu}, \chi\right)=\eta\left(\chi, d x^{\mu}\right)=0$. We shall also assume here that

$$
\eta^{\mu \nu}(x, y)=\theta(y) \tilde{\eta}^{\mu \nu}
$$

where $\tilde{\eta}$ is the standard Minkowski metric and $\theta(y)$ is equal

gammacos$\theta_{W}$ for $y=+$ and $\gamma \sin \theta_{W}$ for $y=-, \theta_{W}$ is the Weinberg angle and $\gamma$ an arbitrary positive number. The metric on the discrete degrees of freedom is determined by one constant:

$$
\eta(\chi, \chi)=\alpha .
$$

After taking such form of the metric the Yang-Mills Lagrange function becomes:

$$
\mathcal{L}_{Y M}(x, y)=\gamma^{2} \theta(y)^{2} F_{\mu \nu} F^{\mu \nu}+\alpha \gamma \theta(y) \mathcal{F}_{\mu}^{\dagger} \mathcal{F}^{\mu}+\alpha^{2}\left(\Psi^{\dagger} \Psi-1\right)^{2}
$$

and the Lagrange function of the bosonic part of electroweak interactions is obtained by taking the sum of the above for $y=+$ and $y=-$.

As can be seen directly in the formula (14) the quartic potential of the Higgs field $\Psi$ has a very special form. Its shape is determined only by one scaling constant, staying in front of the whole term. Therefore one may establish a relation between the mass of the field $\Psi$ and its vacuum expectation value, which contributes to the masses of fields $A_{\mu}$. Consequently, we should expect that the mass of the Higgs field could be determined from the other parameters of Standard Model.

\section{Action term linear in curvature}

In this section we shall present an argument, which implies that the suggested relations do not take place. Moreover, we shall show that the approach based on noncommutative geometry gives us a precise description of the Standard Model, with the same number of free parameters.

Our idea is based on the observation that there exists a possibility of adding an extra gauge invariant term to the Yang-Mills action, which is linear in the curvature $\mathbf{F}$. Let us proceed with the construction.

The curvature two-form $\mathbf{F}$ can be represented as a sum of the tensor product of one forms:

$$
\mathbf{F}=\sum_{i} u_{i} \otimes v_{i}
$$

Although this decomposition is not unique, we may observe that the following quantity does not depend on its choice:

$$
G(\mathbf{F})=\sum_{i} \eta\left(u_{i}, v_{i}\right) .
$$


This is due to the fact that both the tensor product and the metric have the property of linearity. Moreover, this guarantees that $G(\mathbf{F})$ is gauge covariant and therefore its trace remains gauge invariant. If we integrate it, we shall obtain an action, which is linear in curvature:

$$
S_{l}=\int \operatorname{Tr} G(\mathbf{F})+\text { c.c. }
$$

Of course, in the standard differential geometry such action vanishes, because the decomposition (15) is antisymmetrized and the metric is symmetric. However, in the case of noncommutative geometry this no longer holds. In fact, in the model considered in the previous section we have encountered the situation, where the product of two one forms is not antisymmetric: $\chi \otimes \chi \neq 0$. Therefore, in noncommutative geometry this action (17) could be nontrivial.

If we calculate the corresponding Lagrange function in our particular case, the only nontrivial contribution comes from the discrete degrees of freedom:

$$
\mathcal{L}_{l}=4 \alpha\left(\Psi^{\star} \Psi-1\right),
$$

where we have already integrated it over the discrete space $\mathbf{Z}_{2}$. Now, if we add this term to the Yang-Mills action, with an arbitrary scaling parameter $\lambda$, the only change will be in the form of the Higgs potential, which now can be rewritten as:

$$
\mathcal{L}_{\text {Higgs }}=\beta\left(\Psi^{\star} \Psi-\mu\right)^{2},
$$

where $\mu=\frac{\lambda}{2 \beta}-1$. The change of the constant in front of the Higgs potential from $\alpha^{2}$ to $\beta$ reflects that we may as well add the square of (18), thus changing the constant $\alpha^{2}$ to some other arbitrary value $\beta$.

We can see that this is precise form of the Higgs potential with two free parameters, as usually assumed in the Standard Model. Since we see no reason why these terms should be neglected, we may conclude that they could contributes to the total action of the model. Consequently, we obtain the full version of the Standard Model, with no relations between the Higgs mass and other parameters.

\section{Conclusions}

As we have shown in the previous section, the existence of the gauge invariant term linear in the curvature modifies the total action of the Standard Model by increasing its number of free parameters by one, back to the original value. This may be disappointing, as the methods of noncommutative geometry has already raised hopes that the number of free parameters might be reduced. In fact, the indication that the Higgs mass is related to other constants already on the Lagrangian level, was considered as an important result of the noncommutative geometry approach. On the other hand, our observation may be viewed 
as a completion of the derivation of the precise form Standard Model of electroweak interaction from the product of discrete and continuous geometry. It is remarkable that one may obtain exactly the same classical Lagrangian from few geometrical assumptions.

Since we have shown that the most general Lagrangian admits the same number of parameters as the phenomenologically deduced one, we may conclude that there is no specific formula for the Higgs mass. Let us stress this fact that this hold only in case of the assumed discrete geometry being responsible for appearance of Higgses. In other approaches, which use the framework closer to the standard differential geometry [8]-11, the linear term (17) is trivial. Therefore some relations and estimates of the Higgs mass are possible in such cases.

The geometrical description of the electroweak interactions and the spontaneous symmetry breaking mechanism in terms of the product of continuous and discrete geometry is an important step towards our better understanding of these phenomena. Our result that one cannot expect some basic relations between Higgs mass and other parameters suggests that the original Lagrangian corresponds exactly to the one derived by methods of noncommutative geometry. Of course, one cannot exclude that the presented extra terms do not contribute to the total action. This, however, could be verified only by experiment, which would confirm the existence of the Higgs particle and determine its mass.

\section{References}

[1] A.Connes,J.Lott, Particle models and non-commutative geometry, Nucl.Phys. (Proc. Suppl.) B18, (1990) 29-47

[2] A.Connes, Geometrie non Commutative, Intereditions, Paris (1990)

[3] R.Coquereaux, Non-commutative geometry and theoretical physics, Journal of Geometry and Physics, 1990

[4] R.Coquereaux, Non-commutative Geometry: A Physicists Brief Survey, preprint CERN-TH.6522/92

[5] R.Coquereaux,R.Haussling,N.A.Papadopoulos,F.Scheck, Generalized Gauge Transformations and Hidden Symmetry in the Standard Model, Int.J.Mod.Phys. A7, 2809 (1992)

[6] R.Coquereaux,G. Esposito-Farèse, G.Vaillant, Higgs field as Yang-Mills field and discrete symmetries, Nucl. Phys. B353, (1991) 689-706

[7] E.Álvarez, J.M.Gracia-Bondia, C.P.Martin, Parameter restrictions in a non-commutative geometry model do not survive standard quantum corrections, hep-th \# 9302008 
[8] M.Dubois-Violette,J.Madore,R.Kerner, Gauge bosons in noncommutative geometry,

Class.Quant.Grav. 6, (1989) 1709-1724

[9] M.Dubois-Violette,J.Madore,R.Kerner, Phys.Lett. B217, (1989) 485

[10] M.Dubois-Violette,J.Madore,R.Kerner, Non-commutative differential geometry and new models of gauge theory, J.Math.Phys. 31(2), (1990) 323

[11] B.S.Balkrishna,F.Gürsey,K.C.Wali, Noncommutative geometry and Higgs Mechanism in the Standard Model, Phys.Lett. B254, (1991), 430

[12] A.Sitarz, Noncommutative Geometry and Gauge Theory on Discrete Groups, preprint TPJU-7/92, hep-th \# 9210098 